\title{
Distribución y densidad de la trucha Oncorhynchus mykiss (Salmoniformes: Salmonidae) en los Andes venezolanos
}

\author{
Jaime E. Péfaur ${ }^{1} \&$ Nancy M. Sierra ${ }^{1,2}$ \\ Ecología Animal, Facultad de Ciencias, Universidad de Los Andes, Mérida, Venezuela; pefaur@ciens.ula.ve \\ Departamento de Zoologia, I.B. Universidade Estadual de Campinas, Campinas, S.P., Brasil.
}

Recibido 18-IX-1997. Corregido 30-IV-1998. Aceptado 14-IV-1998.

\begin{abstract}
Spatial distribution and relative abundance of wild rainbow trout populations were studied in 68 stations located between 500 and 4000 masl in seven hydrological basins of the most important Andean rivers of Venezuela during a year of sampling. A total of 612 individuals were obtained above $1700 \mathrm{~m}$. Most fishes were obtained from the Chama (43\%), Motatán (21\%) and Santo Domingo (32\%) rivers, very few in the Negro (1.75\%) and La Grita (0.33\%) rivers, and none in the Escalante and Mocotíes rivers basins. Adult males were more abundant than females in a 2.15: 1 proportion. Juveniles made a $56.7 \%$ of captures, with a proportion of 1.30 juveniles per adult. Individual sizes varied from 2.0 to $21.8 \mathrm{~cm}$, most within the interval $5.1-10.0 \mathrm{~cm}$; the weight of specimens also showed an ample variation, with a maximum of $317.0 \mathrm{~g}$.
\end{abstract}

Key words: Rainbow trout, Oncorhynchus mykiss, Andean rivers, hydrological basins, altitudinal distribution.

Entre las especies de mayor valor económico de los Andes venezolanos, se encuentra la trucha arcoiris (Oncorhynchus mykiss), introducida en 1935 (Ginés et al. 1952). Por su extracción y las dificultades para su reproducción, el Ministerio de Agricultura anualmente siembra alevines para afirmar las poblaciones existentes (Sette 1991). Esta reintroducción de alevines y la extracción de adultos, ha hecho difícil estimar las densidades de poblaciones de este pez (Nebiolo 1987, Péfaur 1987, Palencia 1988, Bastardo 1990, Sette 1991, Péfaur y Sierra 1992), así como de sus patrones de distribución en las aguas altoandinas. Los objetivos generales de este trabajo han sido establecer la distribución altitudinal y por cuenca de la especie en los altos Andes venezolanos y estimar la abundancia relativa y proporciones de sexo y edad.

\section{MATERIALES Y METODOS}

Se hizo un reconocimiento biogeográfico de las partes altas de los Estados Trujillo, Mérida, Barinas y Táchira, recorriendo las principales cuencas hidrográficas a objeto de establecer las estaciones de muestreos. Las cuencas estudiadas fueron las de los ríos Chama, Mocotíes, Escalante, La Grita, Negro, Santo Domingo y Motatán, ubicadas dentro del rectángulo de coordenadas $8^{\circ} 10^{\prime}$ y $9^{\circ} 40^{\prime} \mathrm{N}$ y $70^{\circ} 25^{\prime}$ y $72^{\circ} 10^{\prime} \mathrm{W}$.

En cada cuenca se seleccionó un número variable de estaciones de muestreo tanto en quebradas como en sectores del cauce principal del río. El número de estaciones dependió de la accesibilidad al lugar, sobre todo en épocas lluviosas. Se establecieron 68 estaciones de muestreo, entre 
los $500 \mathrm{~m}$ y $4000 \mathrm{~m}$, con un promedio de 9.5 estaciones por cuenca (Cuadro 1). En cada estación se efectuaron hasta tres muestreos a lo largo de 14 meses de trabajo de campo (Enero 1989 a Febrero 1990), con un promedio de 15 muestreos por cuenca (Cuadro 1).

La recolecta de peces se efectuó mediante pesca eléctrica producida por un generador portátil (Nebiolo 1982, 1987, Reynolds 1983) y el uso de una malla con participación de tres o en siete cuencas andinas, fue siempre por encima de los $1700 \mathrm{~m}$. Altitudinalmente, un $9.8 \%$ de las capturas se logrố entre los 1000 y $2000 \mathrm{~m}$, mientras que èl restante $90 \%$ es capturado por encima de los $2000 \mathrm{~m}$ (Cuadro 2).

Las densidades de capturas fueron bastante heterogéneas en las diferentes cuencas. En las recolectas de los ríos Chama, Santo Domingo y Motatán se logró un promedio de alrededor de 11 individuos por muestreo, el cual baja a 5.94

\section{CUADRO 1}

Datos generales de las recolectas de trucha arcoiris en los Andes venezolanos

\begin{tabular}{|c|c|c|c|c|}
\hline Cuenca & $\begin{array}{c}\text { Estaciones } \\
\text { de } \\
\text { muestreos }\end{array}$ & $\begin{array}{l}\text { Número de } \\
\text { muestreos }\end{array}$ & $\begin{array}{l}\text { Individuos } \\
\text { recolectados } \\
\begin{array}{c}\mathrm{N}=612 \\
(\%)\end{array}\end{array}$ & $\begin{array}{c}\text { Promiedio } \\
x \\
\text { muestreo }\end{array}$ \\
\hline Chama & 17 & 21 & 43.14 & 12.57 \\
\hline Santo Domingo & 11 & 18 & 32.03 & 10.88 \\
\hline Motatán & 10 & 13 & 21.08 & 9.92 \\
\hline Negro & 5 & 12 & 3.43 & 1.75 \\
\hline Grita & 7 & 8 & 0.33 & 0.25 \\
\hline Escalante & 6 & 12 & 0.00 & 0.00 \\
\hline Mocotíes & 10 & 19 & 0.00 & 0.00 \\
\hline Total & 66 & 103 & 100.00 & $5: 94$ \\
\hline $\mathrm{X}$ & 9.4 & 14.7 & 87.3 & --- \\
\hline
\end{tabular}

cuatro personas, con un desplazamiento de 80 $\mathrm{m}$ en el curso de agua. Algunos parámetros físico-químicos del agua $\left(\mathrm{O}_{2}, \mathrm{CO}_{2}, \mathrm{~T}^{\mathrm{O}}, \mathrm{pH}\right.$, alcalinidad, cloruros, dureza, estabilidad y conductividad) se midieron in situ y en laboratorio. Los peces recolectados se fijaron en formalina al $10 \%$ durante 8 días, para ser preservados posteriormente en alcohol etílico al $70 \%$ e ingresados a la Colección de Vertebrados de la Universidad de Los Andes (CVULA), Mérida, Venezuela.

\section{RESULTADOS}

El $96 \%$ del total de las truchas fueron recolectadas en las cuencas de los ríos Chama, Santo Domingo y Motatán, mientras que en las cuencas de los ríos Escalante, Mocotíes y La Grita, las capturas fueron escasas o nulas (Cuadro 1). La altura mínima alcanzada por la trucha truchas por muestreo cuando se considera a todas las cuencas evaluadas (Cuadro 1).

El tamaño de las truchas recolectadas presentó un ámbito de variación de $20 \mathrm{~cm}$ aproximadamente, con extremos en los $2.00 \mathrm{~cm}$ y $21.8 \mathrm{~cm}$. Con la finalidad de apreciar la distribución de tamaño de los individuos, se establecieron categorías (longitud standard) con nivel de $5 \mathrm{~cm}$ cada una. Casi la mitad de los especímènes midió entre $5.1-10.0 \mathrm{~cm}$, indicando una franca predominancia de juveniles en las poblaciones (Fig. 1): El peso de los ejemplares también presêntó una amplia variación, con un mínimo de $0.1 \mathrm{~g}$ hasta un máximo de 317.0 g. En los Andes venezolanos es más frecuente hallar truchas de mayor peso en lagunas que en ríos y quebradas (Coché y Taphorn 1995).

Dada la dificultad de establecer el sexo con base en la morfología externa, éste fue determinado por análisis directo de las gónadas. Especímenes menores de $5 \mathrm{~cm}$ automáticamente entraron en la categoría de juveniles indeterminados 
CUADRO 2

Númeres de recolectas de trucha arcoiris distribuidas por cuenca y altitud en los Andes venezolanos

\section{Alturas}

(m)

$500-1000$

1001-2000

2001-3000

Total
3001-4000

\section{Santo}

Chama Domingo

$\begin{array}{rr}0 & 0 \\ 8 & 39 \\ 53 & 117 \\ 203 & 40 \\ 264 & 196\end{array}$

(Cuadro 3). Bastardo (1994) reporta una maduración en ambos sexos a partir de $12 \mathrm{~cm}$ de longitud standard. Los machos adultos superan numéricamente a las hembras en una proporción de 2.15: 1 , sienđo estas diferencias estadísticamente significativas $\left(c^{2}=35.5, p=0.05\right)$. Tanto las categorías de machos como hembras inmaduros superan numéricamente a los machos y hembras maduros, respectivamente. Los juveniles conforman

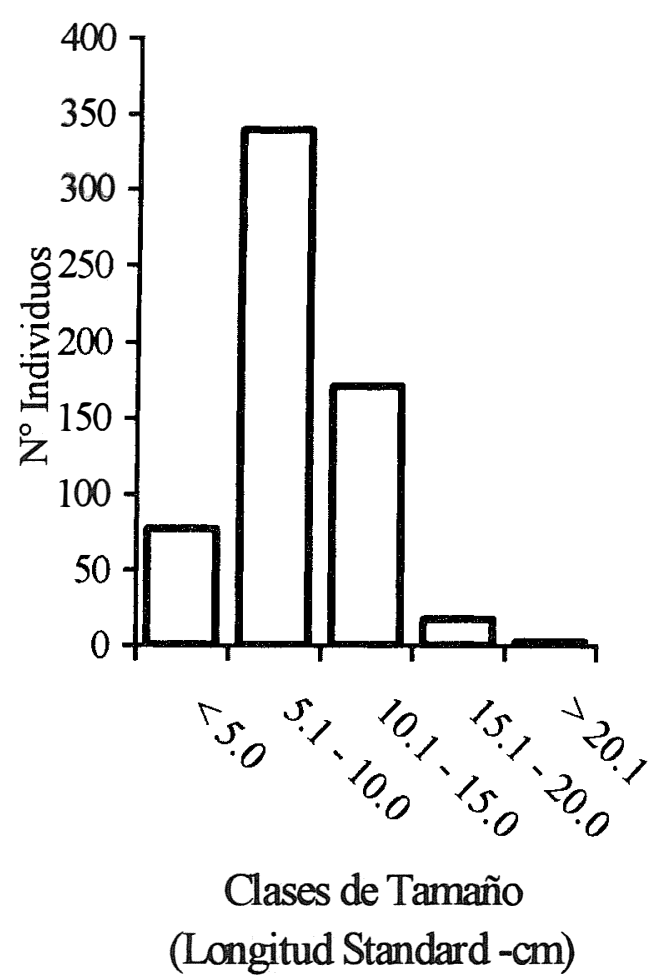

Fig. 1. Frecuencia de las categorías de tamaños (longitud standard) en truchas arcoiris (O. mykiss) recolectadas en los Andes venezolanos.

$\begin{array}{rcccr}\text { Negro } & \text { Grita } & \text { Escalante } & \text { Mocotíes } & \% \\ 0 & 0 & 0 & 0 & 0 \\ 0 & 0 & 0 & 0 & 9.8 \\ 21 & 2 & 0 & 0 & 42.0 \\ 0 & 0 & 0 & 0 & 48.2 \\ 21 & 2 & 0 & 0 & 100.0\end{array}$

un $56.7 \%$ del total de capturas, con una proporción de 1.30 juveniles por adulto, diferencia que es estadísticamente significativa $\left(c^{2}=11.18\right.$; $\mathrm{p}=0.05$ ).

Colectas en las Cuencas de Ríos Andinos.

Río Chama. Este es el río más vasto de toda la zona andina venezolana, con numerosos afluentes; algunos de estos apenas entran en contacto con él, antes de desembocar al Lago de Maracaibo.

$\mathrm{El} \mathrm{pH}$ de las aguas es neutro con leve tendencia hacia la alcalinidad, no alcanzando valores mayores de 7.6. La alcalinidad total presenta fluctuaciones entre estaciones con valores entre $4.3 \mathrm{mg} / \mathrm{l}$ y $119.3 \mathrm{mg} / \mathrm{l}$. Con la excepción de la Quebrada La Caña (2800 m), el bióxido de carbono tiene valores bajos, incluso por debajo de los $1000 \mathrm{~m}$, existiendo una relación inversa entre el contenido de bióxido de

\section{CUADRO 3}

Estado reproductivo de truchas arcoiris capturadas en siete cuencas andinas venezolanas

$\begin{array}{llr}\text { Sexo } & \begin{array}{l}\text { Categoría } \\ \text { reproductiva }\end{array} & \% \\ & \text { Maduros } & 13.40 \\ \text { Machos } & \text { Inmaduros } & 16.17 \\ & \text { Subtotal } & 29.57 \\ & & \\ & \text { Maduras } & 5.06 \\ \text { Hembras } & \text { Inmaduras } & 8.66 \\ & \text { Subtotal } & 13.72 \\ \text { Juveniles Indeterminados } & 56.70\end{array}$

Total 612 Individuos 
carbono y la altura (Péfaur y Durant 1983. Diagnóstico ríos andinos. Act. III Congr. Venez. Conserv. Guanare, Venezuela). El contenido de oxígeno disuelto es favorable para estos peces, con valores $>5 \mathrm{mg} / \mathrm{l}$ en todas las estaciones, excepto en Las González (1200 m) y La Sucia $(1100 \mathrm{~m})$. Previamente, se han señalado valores superiores a $7.0 \mathrm{mg} / \mathrm{l}$ (Hirigoyen 1976. La trucha en Andes venezolanos. Ofc. Nac. Pesca, MAC, Caracas, Venezuela). Resaltan los valores de la dureza total y cálcica en las aguas de La Culata (3000 m), relativamente baja, con valores cercanos a $19 \mathrm{mg} / \mathrm{l}$, al ser contrastadas con 25 a 30 $\mathrm{mg} / \mathrm{l}$ para la misma zona del río (Hirigoyen 1976); otros valores comparativos han sido provistos por Sette (1992).

En esta cuenca fueron extraídos especímenes entre los 1925 y 3540 m (Cuadro 4), con una decreciente frecuencia de mayor a menor altura, y con un alto valor a nivel de los 3540 $\mathrm{m}$, para disminuir entre los 3430 y $2875 \mathrm{~m}$ con valores intermedios. Otro sector distribucional queda ubicado entre los 2800 y $1925 \mathrm{~m}$, donde la captura es baja. Por debajo de estos límites no hubo recolectas.

Río Mocotíes. Aún cuando este río también pertenece a la cuenca del río Chama, su conexión con él está por debajo de los $500 \mathrm{~m}$ y por ello se la consideró como una cuenca aparte. A pesar de haberse realizado 19 muestreos en 10 estaciones, algunas por encima de los $1800 \mathrm{~m}$, en el río Mocotíes no hubo recolectas de truchas. Aquí el oxígeno disuelto alcanza un promedio de $4.70 \mathrm{mg} / \mathrm{l}$, que es el más bajo valor logrado en todas las estaciones estudiadas.

Río Motatán. Las truchas están bien representadas por encima de $2000 \mathrm{~m}$, donde se logró una recolecta de 129 individuos (Cuadro 4). Las aguas de este río tienen el más alto promedio de bióxido de carbono $(0.93 \mathrm{mg} / \mathrm{l})$, valor principalmente influenciado por las estaciones Timotes $(2000 \mathrm{~m})$ y La Puerta $(1800 \mathrm{~m})$, en las cuales sobrepasa los $12 \mathrm{mg} / \mathrm{l}$. La primera se encuentra inmediatamente abajo del pueblo de Timotes, donde la población de truchas es alta. En La Puerta los valores de la alcalinidad total exceden los $70 \mathrm{mg} / \mathrm{l}$, y $164.3 \mathrm{mg} / \mathrm{l}$ de dureza total.

\section{CUADRO 4}

Cantidades de trucha arcoiris recolectadas de acuerdo a las alturas en algunas de las cuencas en estudio*.

Río Chama

$\begin{array}{rcr}\begin{array}{c}\text { Altitud } \\ (\mathrm{m})\end{array} & \begin{array}{c}\text { Individuos } \\ \text { recolectados }\end{array} & \begin{array}{r}\text { Altitu } \\ (\mathrm{m})\end{array} \\ 3540 & 83 & 4000 \\ 3430 & 32 & 2800 \\ 3345 & 17 & 2000 \\ 3245 & 35 & 1800 \\ 3210 & 20 & 1600 \\ 3100 & 16 & 1000 \\ 3000 & 27 & 800 \\ 2875 & 17 & 700 \\ 2800 & 6 & 650 \\ 2130 & 3 & 600 \\ 1925 & 8 & \\ 1770 & 0 & \\ 1400 & 0 & \\ 1200 & 0 & \\ 1100 & 0 & \\ 820 & 0 & \\ 535 & 0 & \\ \text { Totales } & 264 & \end{array}$

Río Motatán

$\begin{gathered}\text { Individuos } \\ \text { recolectados }\end{gathered}$
52
64
13
0
0
0
0
0
0
0

Río Santo Domingo

$\begin{array}{cccc}\begin{array}{c}\text { Altitud } \\ (\mathrm{m})\end{array} & \begin{array}{c}\text { Individuos } \\ \text { recolectado }\end{array} & \begin{array}{c}\text { Altitud } \\ (\mathrm{m})\end{array} & \begin{array}{c}\text { Individuos } \\ \text { recolectados }\end{array} \\ 3105 & 30 & 2010 & 21 \\ 3000 & 10 & 1865 & 0 \\ 2410 & 117 & 1465 & 0 \\ 2210 & 0 & 1395 & 0 \\ 1880 & 2 & 1310 & 0\end{array}$

* Ver Cuadro 1 y Texto. 
Río Santo Domingo. Este es otro de los ríos que se originan en el nudo de Apartaderos que, junto al Chama y el Motatán, conforman la más importante fuente de agua del sector noreste andino venezolano. Tiene, a diferencia de los otros ríos, una represa en la cota de $1500 \mathrm{~m}$ que divide el curso principal de las aguas, dejando dos áreas bien definidas: aquella por encima del espejo de agua, y otras aguas abajo de ésta. En este río se obtuvo la segunda mayor recolecta de truchas, representado un $32 \%$ del total (Cuadro 1). El Baho, a $2410 \mathrm{~m}$, fue la estación que proveyó casi el 60\% del total de capturas (Cuadro 4).

Sin duda que las condiciones físico-químicas de las aguas del Santo Domingo han sido el principal factor del éxito de las truchiculturas establecidas en esta cuenca, y también de la alta densidad de las poblaciones silvestres que allí se encuentran. La alta concentración de oxígeno disuelto, baja presencia de bióxido de carbono, bajo tenor de dureza cálcica, alcalinidad moderada y persistente baja temperatura, hacen de sus aguas las mejores para explotaciones ícticas de aguas frías. Hirigoyen (1976) logró obtener una significativa muestra en recolectas realizadas en la cola del embalse; en contraste, en este estudio no se logró recolectar ninguna en esa localidad $(1780 \mathrm{~m})$, ni frente al Pueblo de Santo Domingo (2210 $\mathrm{m}$ ), y apenas aparecieron dos pequeños alevines en el río Aracay, a $1880 \mathrm{~m}$ (Cuadro 4). Desde los $2200 \mathrm{~m}$ río abajo prácticamente no existe la trucha, correspondiendo estas zonas a sectores agrícolas donde hay un permanente uso de pesticidas. Sin embargo, la estación de 1775 m situada en una quebrada del Parque Nacional Sierra Nevada, se encuentran las segundas mayores abundancias obtenidas en este río.

En la medida que se desciende altitudinalmente, la complejidad de la ictiofauna se incrementa (Taphorn y Lilyestrom 1981, Flecker 1992 a,b). Es a partir de los $800 \mathrm{~m}$ donde se encuentra un cambio drástico en la composición íctica de las comunidades, presentando elementos de neta influencia llanera provenientes de la cuenca del río Apure, como sardinas (Brycon sp.), guabinas (Hoplias malabaricus) y caminadores (Parodon apolinari) (Péfaur y Sierra 1992).
Río Negro. En la mayor parte de su recorrido las aguas del río Negro son lentas a diferencia de los restantes ríos, y con dos cualidades resaltantes: adquieren, en la medida que el río se hace más profundo, un color negro-parduzco y presentan la menor alcalinidad de todas las cuencas ex minadas. Esto hace que en varias partes sea un río con condiciones similares a ríos de zonas bajas.

Sólo en la estación Quebrada Las Truchas $(2010 \mathrm{~m})$ las recolectas fueron positivas (Cuadro 4). Este es, además, el único lugar muestreado en los Andes venezolanos, donde han sido recolectadas truchas junto a otras especies de peces: babositos (Astroblepus sp.) y lauchas (Trichomycterus sp.). Esto es significativo para la actual biogeografía andina, ya que indica que efectivamente había peces autóctonos en los lugares donde hoy sólo existen truchas. Probablemente su introducción llevó a una declinación o extinción de los peces autóctonos (Ginés et al. 1952, Dahl 1971, Taphorn y Lilyestrom 1981, Nebiolo 1987, Péfaur 1987, 1992). Experimentalmente, se ha demostrado el efecto depredador de la trucha arcoiris en la ictiofauna autóctona, con el consiguiente cambio en la conducta y la distribución espacial de las especies predadas (Blinn et al. 1993). Los hábitos alimentarios predatorios oportunistas de la trucha en los Andes venezolanos han sido reportados por Bastardo et al. (1994) y Segnini y Bastardo (1995).

Río La Grita. Este río de la región suroeste de los Andes fue muestreado en siete diferentes estaciones, desde los 540 hasta los 2200 m. Aún cuando los valores de los diferentes parámetros medidos son relativamente homogéneos y con valores intermedios, el agua tiene por característica presentar los más altos valores de alcalinidad total y de dureza total. De esta cuenca sólo se extrajeron dos ejemplares de trucha, en la Estación Las Porqueras, a 2200 m, no lejos de una truchicultura.

Río Escalante. Este río tiene la particularidad de ser el de mayor pendiente de todos los ríos andinos; desde su origen hasta alcanzar la cota de $500 \mathrm{~m}$, hay una distancia aérea directa menor de $50 \mathrm{kms}$ (Péfaur y Durant 1983). Esta 
fuerte inclinación determina muchas de las características biológicas y físico-químicas encontradas en sus aguas. Seguramente debido a su pendiente y rapidez de desplazamiento, sus aguas resultan ser -en promedio- las de menor contenido de bióxido de carbono. Sin embargo, a pesar de haber establecido en su curso cinco estaciones de recolecta y haber muestreado en cinco oportunidades sus aguas, no se logró obtener ni un ejemplar de trucha.

\section{DISCUSION}

La trucha no se encuentra distribuida uniformemente en las aguas de los Andes venezolanos, y por el contrario, presenta fuertes variaciones altitudinales y horizontales en sus tamaños poblacionales. Debido a las introducciones de alevines, esta especie debiera encontrarse en toda esta región biogeográfica, al menos por encima de los $1700 \mathrm{~m}$. Sin embargo, la trucha aparece mayormente en los sectores altos de las cuencas de los ríos asociados al nudo de Apartaderos (Chama, Santo Domingo y Motatán), y minoritariamente en La Grita, pero no se encontró en los ríos Escalante ni Mocotíes.

Con base en los resultados de las recolectas realizadas en siete de las más importantes cuencas de ríos andinos venezolanos, es posible hacer una categorización de ellos estableciendo como criterio las densidades poblacionales de la trucha arcoiris: altas, bajas, o bien ausencia de esta especie. En la primera categoría quedarían incluidas las cuencas de los ríos Chama, Motatán y Santo Domingo; en la segunda, la cuenca del Negro y La Grita; y en la tercera, las cuencas de los ríos Escalante y Mocotíes. Las tres categorías establecidas permiten tener una visión de conjunto de las cuencas de los ríos, para racionalizar el efecto de la pesca, para establecer vedas locales, o bien para orientar las siembras de alevines que anualmente se realizan en estas aguas. Esta categorización servirá también para orientar futuras investigaciones.

Es necesario averiguar los efectos de los parámetros físico-químicos de las aguas de los ríos Escalante y Mocotíes sobre las truchas puesto que si estos ríos son sembrados en una proporción similar que los restantes, la ausencia de truchas ha de deberse a los factores que modifican la calidad del agua. Una razón probable para explicar los bajos tamaños poblacionales o la ausencia de truchas es la contaminación por pesticidas usados en labores agrícolas. $\mathrm{La}$ región andina tiene un alto desarrollo agrícola basado fundamentalmente en el uso de fertilizantes químicos y biocidas, que pueden ser fácilmente arrastrados por las aguas de escorrentia de las laderas hasta los cauces de quebradas o del río. Esta toxicidad de aguas de drenaje agrícola fue letal en cultivos experimentales de Oncorhynchus tschwytscha, así como para otros peces, en California (Saiki et al. 1992).

En cuanto a los tamaños poblacionales, en los Andes venezolanos la trucha presenta poblaciones con densidades fluctuantes e irregulares tanto en sus aspectos altitudinales como temporales. Se trata, sin embargo, de la única especie de los Andes para la cual existe una veda y una temporada de pesca y para la cual se efectúan siembras de alevines. Estas entradas y salidas de individuos a las poblaciones tienen un rol en la demografía de la especie. Los únicos patrones comparativos de los tamaños poblacionales obtenidos previamente son los de Hirigoyen (1976) para los ríos Chama y Santo Domingo, y Nebiolo (1987) y Bastardo (1990) para el río Chama; los resultados presentados son concordantes con los dos últimos autores. El primero, en cambio, presenta valores más altos, que pueden deberse a una mayor extensión del sector del río muestreado en cada oportunidad, o bien a que las extracciones previas al muestreo afectaban menos a las poblaciones, ya que para 1970 - año de sus recolectas - había menor presión de pesca. Hirigoyen (1976) detectó migraciones, lo cual incide en que las poblaciones sean numéricamente inestables.

Desde el punto de vista poblacional, la presencia de un mayor número de juveniles frente a adultos, es un aspecto favorable. Un acúmulo de datos basados en las extracciones durante los períodos de pesca, sería muy deseable para establecer pirámides de edad y de biomasa, a objeto de visualizar el futuro económico de la trucha arcoiris silvestre. 


\section{AGRADECIMIENTOS}

Los autores agradecen a Alba Díaz, Robinson Pérez y Enrique Pereyra, por su estímulo y discusiones a lo largo del estudio y redacción del manuscrito; a Alfredo Correa y Antonio María Castillo por su valiosa ayuda en el trabajo de campo; a Belkis Rivas y Marisela Angelino por la labor secretarial y el manejo de computadores. Esta publicación es parte de los Proyectos PC-108 del CONICIT de Venezuela y C-173-81 del CDCHT-Universidad de Los Andes. Se agradece también a un evaluador anónimo, cuyos comentarios y observaciones permitieron un sustancial incremento de la redacción final del trabajo.

\section{RESUMEN}

En el presente trabajo se informa acerca de la distribución espacial y la abundancia relativa de las poblaciones de la trucha arcoiris silvestre en los Andes Venezolanos, con base en recolectas en 68 estaciones de muestreo durante un año, ubicadas entre los 500 y $4000 \mathrm{msnm}$ en siete cuencas hidrográficas de los ríos más importantes de los Andes de Venezuela. Un total de 612 individuos fueron obtenidos en estaciones por encima de los $1700 \mathrm{~m}$. La mayoría de los peces de recogieron en las cuencas de los ríos Chama (43\%), Motatán (21\%) y Santo Domingo (32\%), con sólo algunos ejemplares recolectados en los ríos Negro (1.75\%) y La Grita $(0-33 \%)$, y con ausencia de capturas en los ríos Escalante y Mocotíes. Los machos adultos superan numéricamente a las hembras en una proporción de 2.15: 1. Los juveniles conforman un $56.7 \%$ del total de capturas, con una proporción de 1.30 juveniles por adulto. El tamaño de los especímenes varió desde 2.0 a $21.8 \mathrm{~cm}$, con casi la mitad de ellos dentro del intervalo $5.1-10.0 \mathrm{~cm}$. El peso de los ejemplares también presentó una amplia variación, con un máximo de $317.0 \mathrm{~g}$

\section{REFERENCIAS}

Bastardo, H. 1990. Ecología de la Trucha Arcoiris, Salmo gairdnieri Richardson, 1836, en una Quebrada del Estado Mérida, Venezuela. Tesis de Maestría, Universidad de Los Andes, Mérida. $169 \mathrm{p}$.

Bastardo, H. 1994. Reproducción y talla media de madurez de la trucha Oncorhynchus mykiss (Salmoniformes: Salmonidae) en los Andes Venezolanos. Rev. Biol. Trop. 42: $263-270$.
Bastardo, H., O. Infante \& S. Segnini. 1994. Hábitos alimenticios de la trucha arcoiris, Oncorhynchus mykiss (Salmoniformes: Salmonidae), en una quebrada altiandina venezolana. Rev. Biol. Trop. $42: 685$ - 693.

Blinn, D. W., C. Runk, D. A. Clark \& J. N. Rinne. 1993. Effects of rainbow trout predation on little Colorado Spinedace. Trans. Amer. Fish. Soc. 122: 139 - 143.

Coché, Z. \& D. Taphorn. 1995. Observaciones preliminares de las poblaciones de Truchas en lagunas de los Andes Merideños. Res. III Congr. Latinoam. Ecología. Mérida, Venezuela. p. 10 - 5 .

Dahl, G. 1971. Los peces del norte de Colombia. INDERENA, Bogotá, Colombia. 391 p.

Flecker, A. S. 1992a. Fish predation and the evolution of invertebrate drift periodicity: evidence from neotropical streams. Ecology 73: 438 - 448.

Flecker, A. S. 1992b. Fish tropical guilds and the structure of a tropical stream: weak direct vs. strong indirect effects. Ecology 73: 927 - 940.

Ginés, H.; G. Marcuzzi \& F. Martin. 1952. Observaciones sobre las condiciones de vida de la trucha en los Andes de Mérida. II Congreso de Ciencias Naturales y Afines, Cuaderno $\mathrm{N}^{\mathrm{O}}$ 3. Venezuela. 48 p. Mem. Soc. Cien. Nat. La Salle (Caracas) 12: 152 - 200.

Nebiolo, E. 1982. Composición de las comunidades de peces de la cuenca hidrológica del río Chama, Mérida, Venezuela. I. El sistema del río Mocotíes. Actas VIII Congr. Latinoam. Zool.: 767 - 780.

Nebiolo, E. 1987. Composición y estructura de la Ictiofauna del río Chama, Mérida, Venezuela. II. Río Chama medio y alto, y Río Mucujún. Bol. Soc. Venezolana Cienc. Nat. 144: $167-184$.

Palencia, P. 1988. Abundancia y diversidad de la ictiofauna de la cuenca alta de los ríos Uribante y Doradas, Táchira, Venezuela. Tesis de Licenciatura. Universidad de Los Andes, Mérida, Venezuela. $183 \mathrm{p}$

Péfaur, J. E. 1987. Fauna de Uribante. I. Estudio de la Ictiofauna de la Cuenca Uribante - Doradas. Informe Convenio ULA -MARNR-CADAFE, Mérida Venezuela. $226 \mathrm{p}$.

Péfaur, J. E. 1992. Catalogación económica de la ictiofauna alto-andina venezolana. Bol. Soc. Cienc. Nat. La Salle (Caracas) 48: 471 - 492.

Péfaur, J. E. \& N. M. Sierra. 1992. Potencial económico de la ictiofauna de los Andes Merideños. Informe Técnico CONICIT (PC-108). 93 p. 
Reynolds, J. 1983. Electrofishing. p. 147 - 163. In L. A. Nielsen \& D. L. Johnson (eds.). Fisheries techniques. Amer. Fish. Soc., Bethesda, Maryland.

Saiki, M. K., M. R. Jennings \& R.. H. Wiedmeyer. 1992. Toxicity of agricultural subsurface drainwater from the San Joaquin Valley, California, to juvenile Chinook salmon and striped bass. Trans. Amer. Fish. Soc. 121: 78-93.

Segnini, S. \& H. Bastardo. 1995. Cambios ontogenéticos en la dieta de la trucha arcoiris (Oncorhynchus mykiss) en un río Andino neotropical. Biotropica 27: 495 - 508.
Sette, S. 1991. Siembra de alevines de trucha en el Estado Mérida. FONAIAP Divulga (34): 2 -5.

Sette, S. 1992. Condiciones hidrográficas del Estado Mérida en relación con la explotación de la trucha Salmo gairdnerii. Veterinaria Tropical 17: 15 - 29.

Taphorn, D. C. \& C. G. Lilyestrom. 1981. Los peces de importancia económica del área Guanare - Masparro. Informe Preliminar. CIDIAT, Mérida, Venezuela. 23 p. 